\title{
Automatización de un generador de luz ultravioleta, controlada con un HMI, de longitud de onda variable
}

\section{Automation of a variable wavelength controlled HMI ultraviolet light \\ generator}

Jorge Luis Yaulema Castañeda. ${ }^{1}$, Paulina Fernanda Bolaños Logroño. ${ }^{2}$, Hector Bryan Mendez Gómez. ${ }^{3}$ \& Valentina Orquera. ${ }^{4}$

Recibido: 14-03-2020 / Revisado: 19-04-2020 /Aceptado: 10-05-2020/ Publicado: 05-06-2020

\begin{abstract}
.
DOI: $\underline{\text { https://doi.org/10.33262/concienciadigital.v3i2.2.1252 }}$

In the present investigation, the main objective was to perform a UV light wavelength control on a prototype, analyzing its optics, the reference of which is the Perkin Elmer 781 spectrophotometer, used in decontamination analysis of surfactant and photodegradation waters, to which the generator has been redesigned, determining the frequency range, reducing the environmental impact, through the implementation of HMI, whose applied methodology, to carry out the automation process whose application approach is determined through the graduation and decontamination photo of the water, presenting a redesign for the generation of UV wavelengths within the electrical and electronic part through Newton's interpolation, reducing the error to $0.05 \%$ least squares of $3.32 \%$, optimizing the control of motors with an HMI connection, using which the generated wavelength is visualized facilitating the navigation and the control of the motors granting to the operator direct interaction with the equipment, obtaining as a result the improvement of the original system, varying the sensors and actuators to obtain wavelengths from $500 \mathrm{~nm}$ to $700 \mathrm{~nm}$, which allows work to be carried out on the decontamination of water and the effectiveness of the system.
\end{abstract}

${ }^{1}$ Escuela Superior Politécnica de Chimborazo, Facultad de Ciencias, Riobamba, Ecuador, jorge.yaulema@espoch.edu.ec

2 Escuela Superior Politécnica de Chimborazo, Facultad de Ciencias, Riobamba, Ecuador, paulina.bolanos@espoch.edu.ec

${ }^{3}$ Universidad de Costa Rica, Facultad de Ciencias. San Pedro, San José, Costa Rica. hector.mendez@ucr.ac.ar

${ }^{4}$ Universidad Nacional de Río Cuarto, Facultad de Ciencias exactas, físico-química y naturales, Río Cuarto, Argentina, vorquera@exa.unrc.edu.ar 
Keywords: Control, Length, Waves, Automation, Ultra violet.

\section{Resumen.}

En la presente investigación, el objetivo principal fue realizar un control de longitudes de onda de luz UV, en un prototipo, analizando su óptica, cuyo referencial es el espectrofotómetro Perkin Elmer 781, usado en análisis de descontaminación de aguas surfactantes y de fotodegradación, para lo cual se ha rediseñado el generador, determinando el rango de frecuencias, disminuyendo el impacto ambiental, mediante la implementación de HMI, cuya metodología aplicada, para efectuar el proceso de automatización cuyo enfoque aplicativo se determina a través de la foto de graduación y descontaminación del agua, presentando un rediseño para la generación de longitudes de onda UV dentro de la parte eléctrica y electrónica a través de la interpolación de Newton disminuyendo el error al $(0.05 \%)$ mínimos cuadrados de (3.32\%) optimizando el control de motores con una conexión en HMI, mediante la cual se visualiza la longitud de onda generada facilitando la navegación y el control de los motores otorgando al operario la interacción directa con el equipo obteniendo como resultado a la mejora del sistema original variando los sensores y actuadores para obtener longitudes de onda de $(500 \mathrm{~nm})$ a $(700 \mathrm{~nm})$ el cual permite desarrollar trabajos en la descontaminación de aguas y efectividad del sistema.

Palabras claves: Longitud, Control, Ondas, Automatización, Ultra violeta.

\section{Introducción}

Los generadores de luz ultravioleta, estudian diferentes efectos o reacciones y la espectroscopía, la cual interviene sobre la materia orgánica. En el país no se realiza la fabricación, construcción o desarrollo de estos equipos los cuales todos son importados al costo definido por el importador dentro del campo Industrial es indispensable disponer de un sistema con características similares para disminuir el impacto ambiental y el daño generado hacia los ecosistemas y el medio ambiente.

Las investigaciones efectuadas, dentro del área específica electro ambiental, se irradia longitudes de onda establecidas mediante las cuales se puede descontaminar el agua, sumados algunos tipos de surfactantes, los tenso activos son los principales elementos y materiales que modifican la calidad del agua, mostrándolas como no aptos para el consumo humano (Paullan, 2015).

Sirtori, López y Rodríguez (2010), Manifiestan que los estudios foto catalíticos no han tenido la importancia debida los cuales han ignorado permanentemente el estudio de la luz y todas las propiedades inmersas dentro del estudio de la materia, las cuales pueden abrir nos muchos campos de estudio entorno a los parámetros de descontaminación de las aguas residuales 
utilizados en las industrias textiles de desechos hospitalarios, petroleras que requieren un análisis íntegro de la composición química de las diferentes muestras.

La presente investigación tiene la finalidad de fabricar un prototipo, el cual esté apto de generar longitudes variables de onda de luz UV, utilizando como referencia un espectrofotómetro descontinuado el cual con las modificaciones pertinentes, se adaptará o construirá un nuevo generador de ondas electromagnéticas alternándolas desde los 500 hasta los $700 \mathrm{~nm}$, generando un equipo competitivo, comparado con los importados generalmente en la industria petrolera, para manejar los elementos tóxicos como son surfactantes fenoles que afectan directamente a las zonas de intervención o explotación

Espectro de radiación electromagnética, Estas ondas electromagnéticas caracterizadas por la composición de su longitud de frecuencia y de onda se clasifican de acuerdo al orden y valoración creciente o decreciente de la misma las cuales representan la totalidad del denominado espectro electromagnético el cual tiene la capacidad de dividirse en varias zonas atendiendo las características más relevantes de todas las radiaciones producidas (Peri, 2015, citado en Yaulema y Rosero 2016).

Espectro visible: constituido, por la radiación total detectada por la vista humana, reflejada entre los $780 \mathrm{~nm}$ hasta $390 \mathrm{~nm}$ de longitudes de onda, o su similar de 3.84 hasta $7.69 \mathrm{~Hz}$ en frecuencia (Peri 2015); A su vez dentro de esta banda se subdividen diferentes secciones identificadas por las coloraciones como luz blanca mezclada de cierta manera con toda la cromática del respecto. las estrellas y el sol constituyen una fuente directa de luz ultravioleta la cual es visible utilizada en las diferentes lámparas para realizar la iluminación artificial (Requena y Zúñiga 2004).

Radiación electromagnética: Peri (2015), Dentro de su investigación manifiesta que la electroscopia, se establece con la radiación y la intervención de las sustancias orgánicas e inorgánicas como la atribución directa a la longitud de onda ( $\lambda$ ) (Skoog, D 2008). Si en este marco de estudio está comprendida una variabilidad de medidas en función de la frecuencia y longitud de las ondas, recalcando las interacciones con las partículas de radiación respondiendo directamente a un campo alternante con la presencia de una frecuencia variante (v) (Requena y Zúñiga, 2004).

Rayos X y cósmicos: constituidas por las ionizaciones de las moléculas, UV visibles, distribuidas como transiciones electrónicas entre los orbitales moleculares y atómicos, los infrarrojos constituyen las deformaciones entre los enlaces químicos, los microondas reflejan la rotación entre los enlaces químicos y la radiofrecuencia constituida en la transición de Espín electrónico en los átomos de las moléculas (Skoog, Holler y Crouch, 2008). 
La electroscopía UV: Su campo es desarrollado en la propagación electromagnética abarcando desde los $100-700 \mathrm{~nm}$ de longitud de onda los mismos que representan el rango e intervalo de visibilidad, por lo cual este espectro representa la interacción y respuesta del sistema en función directa de la longitud de onda o de frecuencia (Córdoba, 2009, citado en Yaulema y Rosero 2016).

Dentro de los rangos visibles y aproximados a los infrarrojos un espectro electromagnético utiliza la longitud de onda la que a su vez produce el máximo de absorbancia conocida como $\Lambda$ máx, el cual facilita la filtración y la propagación de la luz ultravioleta visible de un cuerpo a otro, proyectando que un electrón desde un estado fundamental (EF), salte directamente a un paso de excitación (EE), eliminando por completo la sobrecarga generada energía representada en calor (Granada, 2015).

Este campo es específico para el estudio del comportamiento de la luz a través de la interacción de modelos matemáticos, analizados y determinados mediante la luminosidad desde el punto de vista propagativo de energía radiante los cuales comprenden 3 principios fundamentales cómo son la propagación directa de la luz la trayectoria de los rayos luminosos y el principio de Fermat comprendido en el periodo de tiempo que la luz necesita recorrer un distanciamiento entre dos puntos, calculado como el máximo y mínimo (Viticodoc, 2010).

Longitud de onda: se basa en los principios de perturbación directa, Cuya base cognitiva es referenciada por una naturaleza diversa con una particularidad de propagación directa, la longitud de onda es precisada como intervalo sucesivo entre dos puntos, comportándose en un mismo periodo de tiempo con una ruta de desplazamiento ondulatoria entre 2 crestas, Resultando que la radiación UV, está en un arreglo inversamente proporcional a la frecuencia la cual comprende los 300 y $700 \mathrm{~nm}$ (Skoog, 2008).

Sistema de espectrofotómetro: Característico en los procesos de espectrofotometría ultravioleta de luz visible, este sistema registra las longitudes de onda realizando una medida comparativa entre la radiación absorbida por una solución midiendo también la intensidad de la luz qué pasa a través de una muestra (I), comparando directamente con la intensidad de luz antes de realizar su paso a través de la muestra (Io) (Harris, 2006).

El equipo utilizado para medir la cantidad de luz qué absorben las muestras constituidas por un equipamiento con filtros de interferencia es a través del espectrofotómetro, según Skoog (2008), manifiesta que este instrumento tiene la funcionalidad de permitir el paso de un rayo de luz a través de las muestras principales para medir la intensidad de la luz con que llega al sensor el cual generará una nueva longitud de onda con la ayuda de los tubos fotomultiplicadores (Skoog, Holler y Crouch 2008). 
ISSN: 2600-5859

Con estas particularidades la fuente luminosa representada por el espectrofotómetro Perkin Elmer 781, utiliza una lámpara de tungsteno la cual ópera con $2.5 \mathrm{~V}$, mediante la cual genera unas que atraviesan los espejos, rendijas y filtros, hasta llegar al monocromador el cual por sus propiedades y funcionalidad divide la luz blanca en los colores del espectro representadas por las diferentes longitud de onda, en su parte final posee un lente reflector cóncavo en el cual se encuentra el sensor de luz ultravioleta visible, este sistema tiene la particularidad de medir la absorbancia de cada uno de los procesos (Pérez y Rengifo, 2011).

Zúñiga (2004) manifiesta que A través del proceso combinado de las bandas de absorción de los infrarrojos conformado por las transiciones electrónicas de bajo consumo la cual inicialmente fue concebida como una zona demasiado compleja para su análisis investigación e interpretación por lo cual fue mal utilizada y valorizada; a través de los avances en cuanto a la óptica geométrica, técnicas químicas y generación de aspectos complejos han dado el uso adecuado de NIR (espectroscopia del infrarrojo más cercano), como intervención dentro de las industrias de alimentación, farmacéuticos, química y un paso agigantado dentro de la agricultura (Elmer, 2004).

El uso y aplicación de NIR, dentro de las investigaciones y valoraciones en un laboratorio clínico constituye una técnica simple y rápido capaz de analizar con precisión muestras biológicas para determinar con exactitud un modelo y método para derivar los resultados del NIR. estos análisis se inician con una mínima preparación de las muestras y reactivos describiendo secuencialmente los procesos de adquisición de datos análisis calibración mediante la utilización de proteínas triglicéridos y glucosas (Elmer, 2004).

Dentro del análisis y representación de los datos obtenidos por la aplicación de esta técnica y equipo se dan por las gráficas generando un informe de los resultados obtenidos los cuales facultan y validan mediante las investigaciones realizadas por, Garcés, Mejía y Santamaría (2011), los cuales Adicionalmente aportan una serie de argumentos para el funcionamiento expresados a continuación:

- Los elementos principales para que exista un buen funcionamiento de estos motores son un rotor y sobre él las adaptaciones de imanes permanentes junto con devanados en el estator.

- Se debe considerar la permutación de estos elementos, dadas por un controlador programable el cual es encargado de enviar constantemente las diferentes variantes de las señales los cuales accionarán las bobinas en el estator generador de movimientos, representando a su vez el papel directo del rotor basado en el imán permanente que lo constituye (Todorobot, 2016). 


\section{Metodología}

La metodología seleccionada para esta investigación, es de tipo aplicativa la cual comprende la generación del proceso de automatización, de una lámpara con variabilidad de longitudes de onda ultravioleta, la cual va a ser interactuada mediante la interfaz hombre máquina (HMI), cuyo enfoque aplicativo está basado en la foto de graduación y la descontaminación de aguas que por lo general generan un problema mayor las que contienen surfactantes, generando así el proceso investigativo mediante la inducción y fortalecimiento al conocimiento sobre el desempeño y funcionamiento de este sistema de desinfección.

Como siguiente paso, se determinaron varios procedimientos, estableciendo de manera inicial, La Constitución del diseño del sistema electrónico y eléctrico, determinado por la variación del control de motores para el encendido de la lámpara de tungsteno, proceso que se realizará a través de un PLC, y obtener la conexión de un HMI, en el manejo de varios autómatas, comprendidos entre los motores de paso el motor AC, los sensores y la lámpara de todo el sistema.

Una vez generados todos estos componentes, se procedió a establecer la estructura física del prototipo, diseñando la base los soportes calculados con todos los pesos y materiales a utilizar la carcasa protectora la cual por investigaciones y recomendaciones se ha determinado como un color opaco o negro la cual es capaz de impedir el ingreso o salida de la luz, cuyo objetivo es precautelar la conservación de todas las muestras, para pasar con el rediseño del sistema electromecánico referido al monocromador y la rendija que constituyen los elementos clave en la descomposición de la luz obtenida a partir de la lámpara y sus diferentes longitudes de onda.

Como aporte final dentro del proceso de generación del prototipo se define la programación del sistema mediante la configuración total de los PLC, y la realización del diseño de la interfaz del HMI, para generar el prototipo con su diseño que a su vez entrará en un análisis método lógico analítico estadístico sobre las pruebas de las longitudes de ondas generadas utilizando varios métodos como el de la regresión lineal de los mínimos cuadrados y la interpolación de Newton el desarrollo de la ecuación del error y la verificación por medio de la varianza ANOVA.

\section{Resultados}

Para efectuar el proceso de análisis de la longitud de onda mediante la aplicación de los métodos de ajuste se realizó el análisis de varianza representado en la tabla 1. 
Tabla 1. Análisis de varianza de las longitudes de onda

\begin{tabular}{cccccc}
\hline Parámetros & $\begin{array}{c}\text { Grados de } \\
\text { libertad }\end{array}$ & $\begin{array}{c}\text { Suma de } \\
\text { cuadrados }\end{array}$ & $\begin{array}{c}\text { Media } \\
\text { Cuadrado }\end{array}$ & Valor F & $\operatorname{Pr}(>\mathrm{F})$ \\
\hline Métodos & 2 & $1.91 \mathrm{E}-15$ & $9.57 \mathrm{E}-16$ & 0.0735 & 0.9292 \\
Residuos & 117 & $1.52 \mathrm{E}-12$ & $1.30 \mathrm{E}-14$ & & \\
\hline
\end{tabular}

Fuente: Elaboración propia.

Dentro de este análisis de varianza podemos observar que el valor de la probabilidad es de 0.9292, por lo cual no existe una diferencia significativa entre la metodología utilizada, llegando a la conclusión qué se puede utilizar el método de los mínimos cuadrados, como también el de interpolación de Newton, con la finalidad de comprobar y respaldar los resultados se utilizó la prueba de Duncan expresados en la tabla 2.

Tabla 2. Análisis de métodos estadísticos

\begin{tabular}{ccc}
\hline Método & Media & Rango \\
\hline $\begin{array}{c}\text { M. Teórico } \\
\text { M. }\end{array}$ & $5.43 \mathrm{E}-07$ & $\mathrm{a}$ \\
$\begin{array}{c}\text { Interpolación } \\
\text { M. Mínimo }\end{array}$ & $5.43 \mathrm{E}-07$ & $\mathrm{a}$ \\
$\begin{array}{c}\text { cuadrados } \\
\text { Letras iguales no difieren }\end{array}$ & $5.35 \mathrm{E}-07$ & $\mathrm{a}$ \\
\multicolumn{3}{c}{ significativamente con $\alpha=0.05$} \\
\hline
\end{tabular}

Fuente: Elaboración propia.

La comprobación estadística mediante el test de Duncan, ratificaron todos los resultados obtenidos en el análisis de varianza comparando estadísticamente las longitudes de onda, cualquier método escogido es factible para ser representado dentro del HMI, encontrando en la representación gráfica que un método tiene un menor error, el cual realizando este proceso se procedió a determinar el mejor método cómo podemos observar en la tabla 3.

Tabla 3. Análisis de la varianza del error de los métodos estadísticos

\begin{tabular}{lrlllll}
\hline Parámetro & $\begin{array}{l}\text { Grados de } \\
\text { Libertad }\end{array}$ & $\begin{array}{l}\text { Suma de } \\
\text { Cuadrados }\end{array}$ & $\begin{array}{l}\text { Media } \\
\text { Cuadrada }\end{array}$ & Valor F & $\operatorname{Pr}(>\mathrm{F})$ & Significancia \\
\hline Métodos & 1 & 219.52 & 219.52 & 52.14 & $2.99 \mathrm{E}-10$ & $* * *$ \\
Residuos & 78 & 328.39 & 4.21 & & & \\
Significancia: $0:^{* * *} ; 0.001:{ }^{* *} ; 0.01:^{*}$ & & & & \\
\hline
\end{tabular}

Fuente: Elaboración propia. 
Desarrollando el análisis ANOVA podemos observar que el valor de la probabilidad es de 2.99 E-10, mostrando una diferencia significativa de entre los métodos utilizados cómo podemos observar en la tabla 4

Tabla 4. Análisis estadístico del error

\begin{tabular}{|c|c|c|}
\hline Método & Media & Rango \\
\hline $\begin{array}{r}\text { Error M. } \\
\text { Mínimos } \\
\text { Cuadrados }\end{array}$ & 332.325 & $A$ \\
\hline Error M. & 0.1025 & $B$ \\
\hline \multicolumn{3}{|c|}{$\begin{array}{l}\text { Letras iguales no difieren } \\
\text { significativamente con } \alpha=0.05\end{array}$} \\
\hline
\end{tabular}

Fuente: Elaboración propia.

del análisis desde diseño del dispositivo el cual estaba establecido para medir la longitud de onda, se utilizó el sensor digital RGB, el cual permite diferenciar los aspectos electromagnéticos producidos por el haz de la luz, encontrando que la energía recibida en la etapa final es baja motivo por el cual se concluye que el sensor RGB no arroja la información de los aspectos electromagnéticos, motivo por el cual se procedió a realizar una aproximación matemática para representar dentro del sistema y poder determinar la longitud de onda del HMI, conociendo explícitamente que estos sistemas deben cumplir con las condiciones de los valores teóricos los cuales proporcionan diferentes fórmulas físicas de longitud de onda representadas por la siguiente determinante:

$$
\lambda=\frac{c}{f}
$$

el prototipo ópera dentro de los rangos de longitud de 500 hasta los $700 \mathrm{~nm}$, generando una gráfica de frecuencia en función de la longitud de onda cómo se puede evidenciar en la figura 1.

Figura 1. Ecuación Original

Ecuación Original

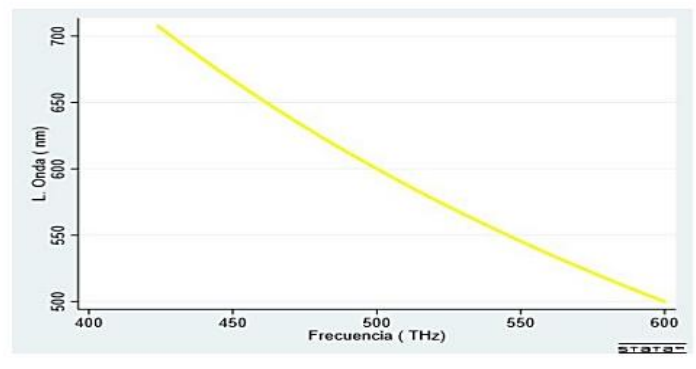

Fuente: Elaboración propia. 
Se utilizó los métodos planteados estadísticamente para ajustar e identificar a los resultados obtenidos reflejados en la regresión lineal a través de los mínimos cuadrados y la interpolación de Newton.

El método de os mínimos cuadrados: Utilizado para ajustar los valores obtenidos dentro de las medias geométricas reflejados por la frecuencia de los espectros electromagnéticos de acuerdo a los valores teóricos estos puntos generan una ecuación la cual satisface a todo el sistema expresadas en la tabla 5.

$$
\lambda=a_{0}+a_{1} f
$$

Tabla 5. Cálculos de los valores de mínimos cuadrados

\begin{tabular}{cc}
\hline \multicolumn{2}{r}{ VALORES OBTENIDOS POR } \\
EL MÉTODO DE LOS MÍNIMOS \\
CUADRADOS \\
$\Sigma x i$ & $2.69 \mathrm{E}+15$ \\
$\Sigma y i$ & $2.86 \mathrm{E}-06$ \\
$\mathrm{~N}$ & 5 \\
$\Sigma x i y i$ & $1.50 \mathrm{E}+09$ \\
$\Sigma x i 2$ & $1.48 \mathrm{E}+30$ \\
$a 0$ & $1.10 \mathrm{E}-06$ \\
$a 1$ & $-9.84 \mathrm{E}-22$ \\
\hline
\end{tabular}

Fuente: Elaboración propia.

Reemplazando estos datos dentro de la ecuación se obtiene que:

$$
\lambda=1.10 \mathrm{E}-06-9.75 \mathrm{E}-22 * \mathrm{f}
$$

Sí ejecutando este reemplazo en la ecuación con los valores de la frecuencia para obtener la siguiente gráfica expresada en la figura 2.

Figura 2. Longitud de Onda vs. Frecuencia

Método de los Mínimos Cuadrados

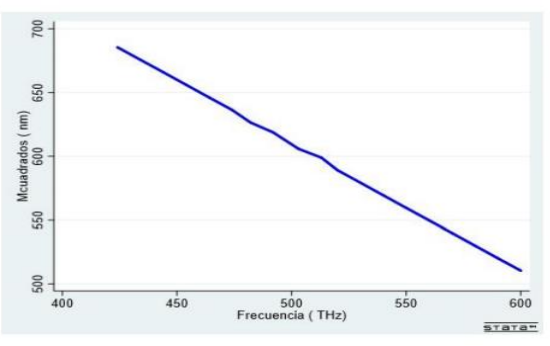

Fuente: Elaboración propia. 
Con la comparación de las gráficas obtenidas en la figura 1 y figura 2, se puede evidenciar que no siguen un mismo camino, deduciendo a su vez que este método no es el que mejor aproximación otorga, el mismo se encuentra representado y graficado en la figura 3.

Figura 3. Comparación Método de Cuadrados Y Ecuación Original

Gráfica longitud de onda vs frecuencia

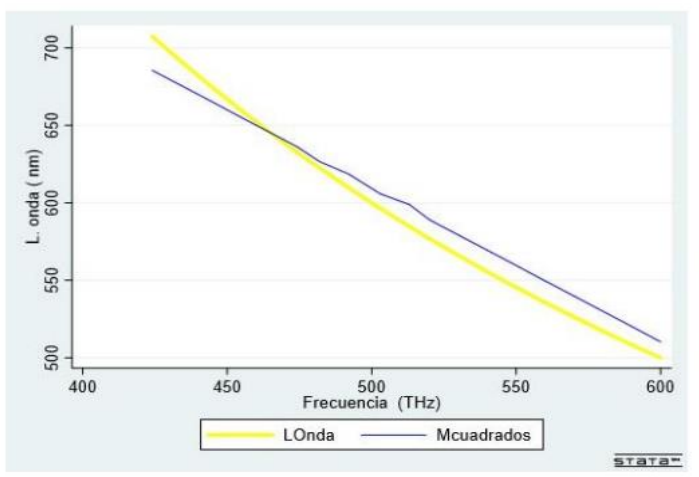

Fuente: Elaboración propia.

Método de interpolación de Newton: este método, al igual que el método de los mínimos cuadrados nos faculta generar una ecuación que satisfaga a todo el sistema aplicando la siguiente ecuación:

$$
\begin{gathered}
\lambda=b_{0}+b_{1}\left(f-f_{0}\right)+b_{2}\left(f-f_{0}\right)\left(f-f_{1}\right)+b_{3}\left(f-f_{0}\right)\left(f-f_{1}\right)\left(f-f_{2}\right) \\
+b_{4}\left(f-f_{0}\right)\left(f-f_{1}\right)\left(f-f_{2}\right)\left(f-f_{3}\right)+b_{5}\left(f-f_{0}\right)\left(f-f_{1}\right)\left(f-f_{2}\right)\left(f-f_{3}\right)\left(f-f_{4}\right)
\end{gathered}
$$

Para la consecución de los valores del índice se aplicó las fórmulas de interpolación obteniendo los datos representados en la tabla 5, para lo cual se reemplazó estos datos en la ecuación anterior obteniendo los siguientes valores:

$$
\begin{aligned}
\lambda=4.21 \mathrm{E}-07 & -6.63 \mathrm{E}-22(\mathrm{f}-7.13 \mathrm{E}+14)+1.17 \mathrm{E}-36(\mathrm{f}-7.13 \mathrm{E}+14)(\mathrm{f}-6.35 \mathrm{E}+14) \\
& -2.32 \mathrm{E}-51(\mathrm{f}-7.13 \mathrm{E}+14)(\mathrm{f}-6.35 \mathrm{E}+14)(\mathrm{f}-5.64 \mathrm{E}+14)+4.73 \mathrm{E} \\
& -66(\mathrm{f}-7.13 \mathrm{E}+14)(\mathrm{f}-6.35 \mathrm{E}+14)(\mathrm{f}-5.64 \mathrm{E}+14)(f-5.06 \mathrm{E}+14) \\
& -1.10 \mathrm{E} \\
& -80(\mathrm{f}-7.13 \mathrm{E}+14)(\mathrm{f}-6.35 \mathrm{E}+14)(\mathrm{f}-5.64 \mathrm{E}+14)(f-5.06 \mathrm{E}+14)(f \\
& -4.92 \mathrm{E}+14)
\end{aligned}
$$


Generando un reemplazo significativo de los datos en la ecuación obtenida para obtener la siguiente gráfica y valoración del método de interpolación de Newton evidenciado como la longitud de onda versus la frecuencia cómo se muestra en la figura 4

Figura 4. Longitud de onda Vs. Frecuencia

Método de Interpolación de Newton

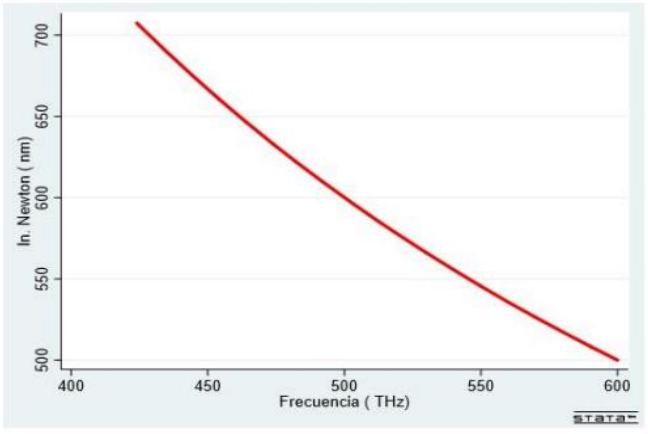

Fuente: Elaboración propia.

En el análisis de comparación de las gráficas obtenidas en la figura 1 y figura 4 se genera hice analiza dentro de la figura 5, apreciando directamente las curvas, las cuales generan una secuencia similar a la original, lo cual nos permite deducir qué es la mejor que se aproxima al método y nos faculta el proceso de cálculo del error de ambos casos.

Figura 5. Comparación del método de Newton Vs. Ecuación Original

Grafica longitud de onda vs frecuencia

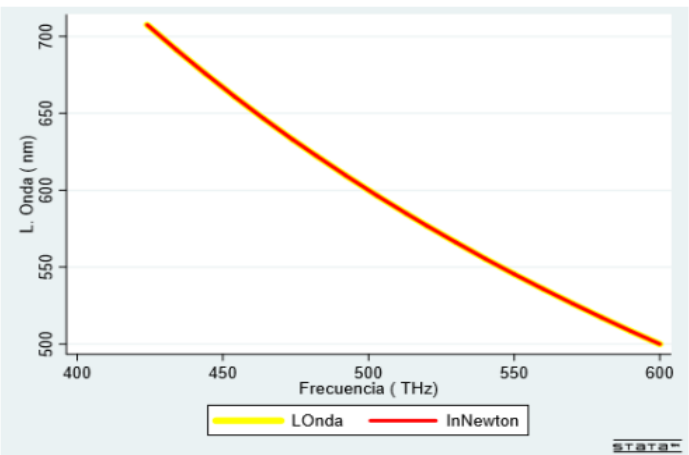

Fuente: Elaboración propia.

Para el cálculo del error el cual se generó a través del uso de cada método aplicamos la siguiente ecuación: 
ISSN: 2600-5859

Vol. 3, N².2, p. 132-153, junio, 2020

$$
\text { Error }=\frac{\lambda_{\text {teorico }}-\lambda \text { ajuste }}{\lambda_{\text {teorico }}} * 100
$$

Tabla 6. Valores obtenidos y análisis de error

\begin{tabular}{|c|c|c|c|c|}
\hline \multicolumn{3}{|c|}{ VALORES OBTENIDOS } & \multicolumn{2}{|c|}{ ANALISIS DE ERROR } \\
\hline L. DE ONDA & M.CUADRADOS & INTERPOLACION & ERROR M.C.\% & ERROR IN. \% \\
\hline 3.90E-07 & 3.44E-07 & $3.90 \mathrm{E}-07$ & 11.83 & 0.14 \\
\hline 3.95E-07 & $3.54 \mathrm{E}-07$ & 3.95E-07 & 10.48 & 0.09 \\
\hline $4.01 \mathrm{E}-07$ & 3.64E-07 & 4.00E-07 & 9.20 & 0.06 \\
\hline 4.06E-07 & 3.74E-07 & 4.06E-07 & 7.99 & 0.03 \\
\hline $4.12 \mathrm{E}-07$ & 3.83E-07 & $4.11 \mathrm{E}-07$ & 6.84 & 0.01 \\
\hline 4.17E-07 & 3.93E-07 & 4.17E-07 & 5.76 & 0.00 \\
\hline 4.23E-07 & 4.03E-07 & 4.23E-07 & 4.75 & 0.00 \\
\hline $4.29 \mathrm{E}-07$ & 4.13E-07 & $4.29 \mathrm{E}-07$ & 3.80 & 0.00 \\
\hline 4.35E-07 & 4.23E-07 & 4.35E-07 & 2.91 & 0.01 \\
\hline 4.42E-07 & 4.33E-07 & 4.42E-07 & 2.09 & 0.01 \\
\hline 4.48E-07 & 4.42E-07 & 4.48E-07 & 1.34 & 0.00 \\
\hline $4.55 \mathrm{E}-07$ & 4.52E-07 & 4.55E-07 & 0.65 & 0.00 \\
\hline 4.69E-07 & 4.71E-07 & 4.69E-07 & 0.47 & 0.00 \\
\hline 4.76E-07 & 4.81E-07 & 4.76E-07 & 0.97 & 0.00 \\
\hline 4.84E-07 & 4.91E-07 & 4.84E-07 & 1.40 & 0.00 \\
\hline 4.92E-07 & $5.00 \mathrm{E}-07$ & 4.92E-07 & 1.77 & 0.00 \\
\hline $5.00 \mathrm{E}-07$ & 5.10E-07 & $5.00 \mathrm{E}-07$ & 2.07 & 0.00 \\
\hline $5.08 \mathrm{E}-07$ & $5.20 \mathrm{E}-07$ & $5.08 \mathrm{E}-07$ & 2.30 & 0.00 \\
\hline 5.17E-07 & 5.30E-07 & 5.17E-07 & 2.47 & 0.00 \\
\hline $5.26 \mathrm{E}-07$ & $5.40 \mathrm{E}-07$ & $5.26 \mathrm{E}-07$ & 2.58 & 0.00 \\
\hline 5.36E-07 & 5.50E-07 & 5.36E-07 & 2.61 & 0.00 \\
\hline $5.45 \mathrm{E}-07$ & 5.60E-07 & $5.45 \mathrm{E}-07$ & 2.59 & 0.00 \\
\hline 5.56E-07 & 5.69E-07 & 5.56E-07 & 2.49 & 0.00 \\
\hline 5.66E-07 & 5.79E-07 & 5.66E-07 & 2.33 & 0.00 \\
\hline 5.77E-07 & 5.89E-07 & 5.77E-07 & 2.11 & 0.00 \\
\hline 5.88E-07 & 5.99E-07 & 5.85E-07 & 1.82 & 0.00 \\
\hline 5.96E-07 & 6.06E-07 & 5.96E-07 & 1.58 & 0.00 \\
\hline $6.12 \mathrm{E}-07$ & 6.19E-07 & $6.10 \mathrm{E}-07$ & 1.04 & 0.00 \\
\hline $6.22 \mathrm{E}-07$ & $6.26 \mathrm{E}-07$ & $6.22 \mathrm{E}-07$ & 0.66 & 0.00 \\
\hline 6.36E-07 & 6.36E-07 & 6.33E-07 & 0.12 & 0.00 \\
\hline 6.49E-07 & $6.46 \mathrm{E}-07$ & $6.47 \mathrm{E}-07$ & 0.49 & 0.00 \\
\hline $6.64 \mathrm{E}-07$ & $6.56 \mathrm{E}-07$ & $6.61 \mathrm{E}-07$ & 1.16 & 0.00 \\
\hline 6.79E-07 & 6.66E-07 & $6.76 \mathrm{E}-07$ & 1.89 & 0.00 \\
\hline 6.94E-07 & $6.76 \mathrm{E}-07$ & 6.91E-07 & 2.70 & 0.00 \\
\hline 7.11E-07 & $6.86 \mathrm{E}-07$ & 7.08E-07 & 3.56 & 0.00 \\
\hline 7.28E-07 & $6.95 \mathrm{E}-07$ & $7.25 \mathrm{E}-07$ & 4.50 & 0.00 \\
\hline 7.46E-07 & 7.05E-07 & 7.42E-07 & 5.50 & 0.01 \\
\hline 7.65E-07 & 7.15E-07 & 7.61E-07 & 6.56 & 0.02 \\
\hline 7.81E-07 & 7.23E-07 & 7.81E-07 & 7.46 & 0.03 \\
\hline
\end{tabular}

Fuente: Elaboración propia 


\section{Diseño y construcción del sistema eléctrico y electrónico.}

Figura 6 Diagrama Funcionamiento sistema

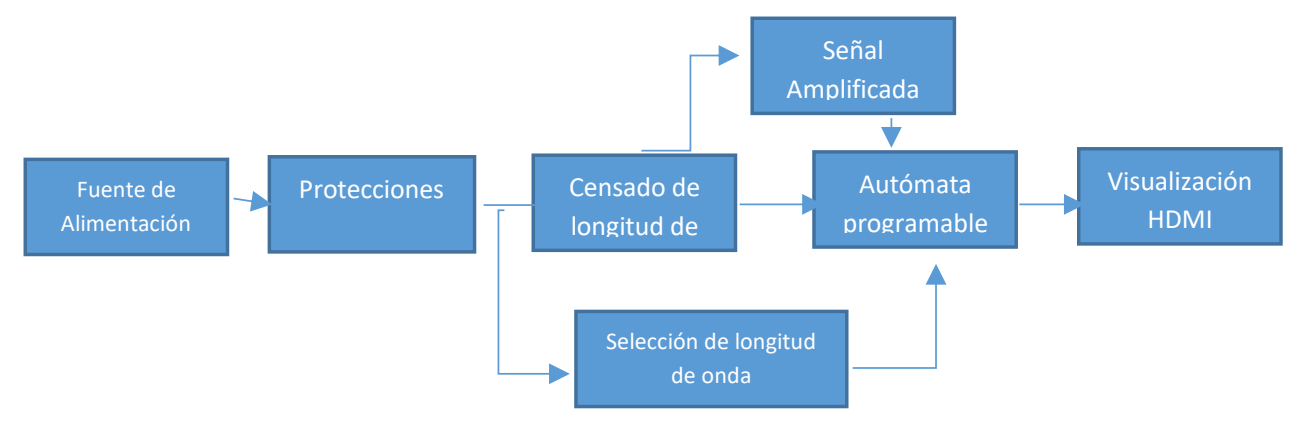

Fuente: Elaboración propia

Los elementos usados para elaborar esta aplicación electrónica se encuentran determinados por el controlador lógico programable (PLC), una pantalla táctil para el interfaz humano maquina (HMI) motores tipo switch, circuitos, lámpara de tungsteno, Enfocado hasta el monocromador, y su transformador, el cual provee a la lámpara un voltaje de $2.5 \mathrm{~V}$ y una corriente característica de 2 A.

\section{Funcionamiento del sistema electrónico y eléctrico del equipo}

Durante el proceso de ponerlo en marcha y energizar al equipo el encendido responde directamente a la pantalla táctil, Interfaz Humano Maquina (HMI), el cual genera un despliegue de un menú mostrando diferentes longitudes de onda procesadas atreve a través del PLC (Yaulema y Rosero 2016).

El sistema de automatización, integró comprende el control del motor AC, los motores a pasos, lámparas y sensores, encargándose de la funcionabilidad del monocromador y la rendija, los cuales descomponen la luz obtenida a partir de la lámpara en las diferentes longitudes de onda, dentro de este proceso el motor AC, se activa el filtro del chopeo, generando a su vez una señal más fina para su medición, controladas por el autómata el cual genera diferentes valores arrojados por los sensores y las variables de la pantalla Touch encargados de enviar las señales para el funcionamiento y movimiento de los motores y la lectura de los valores de longitud de onda en el HMI (Yaulema y Rosero 2016).

PLC Allen Bradley Micro820: Representado en la figura 7, está conformado con Ethernet integrado puertos en serie y una ranura micro SD, posee además una entrada y salida de los registros de datos analógicas esenciales para la automatización remota (Rockwellautomation, 2014). 
Figura 7 PLC Micro 820

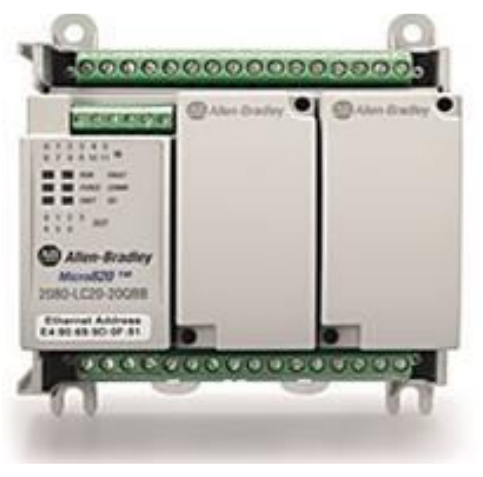

Fuente: Elaboración Propia

Diseño electrónico del sistema: costa del rediseño de todos los circuitos electrónicos originales, controlados por los microcontroladores los cuales son responsables del funcionamiento de los motores AC y DC, Figura 8, modificando circuitos, permitiendo el acoplamiento de control desde PLC y HMI optimizando el movimiento de estos motores a un solo control total, su tamaño de los circuitos, mediante el programa Proteus, simulando y creando distintas placas, Figura 9 (Yaulema y Rosero 2016).

Figura. 8 Tarjetas de control del sistema original

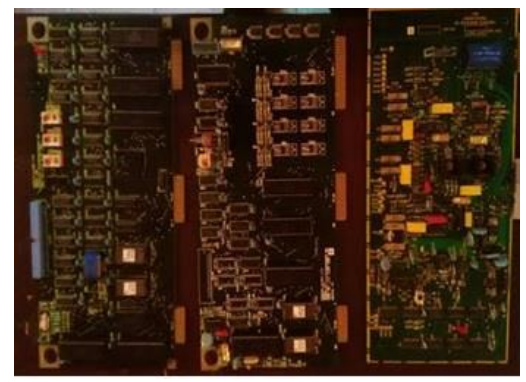

Figura. 9 Circuitos de control rediseñados

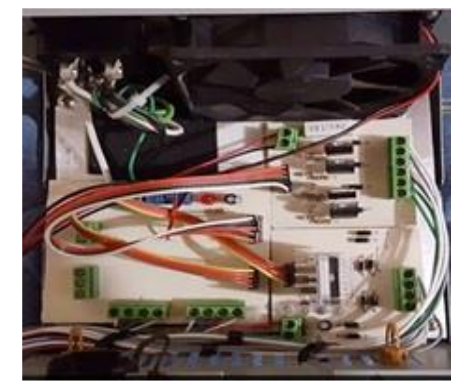

Fuente: Elaboración propia

Diseño de control AC, para el filtro "Chopper": la conformación y diseño de la base permite el control de los disparos en un tiristor, Generando un tren de pulsos, y cierto ángulo de retardo del disparo, conocido como $\alpha$, respecto al paso de cero, por las tensiones alternas las cuales son encargadas de alimentar el convertidor, generando una señal de referencia sinusoidal, conocida también como señal de diente de Sierra sincronizada con la red alterna de alimentación global (Yaulema y Rosero 2016). 
Primera Etapa: Cruce por cero: La señal de referencia dada por la red, realiza una sincronización, la cual utiliza una rectificadora de onda completa generando una señal DC, obteniendo como característica, la pulsación qué es detectada por un optoacoplador PC817, que al momento de apagarse el led interno, genera una reacción, constituida por un pulso, representada en la figura 10 y 11 (Yaulema y Rosero 2016).

Figura. 10 Diagrama de Circuito

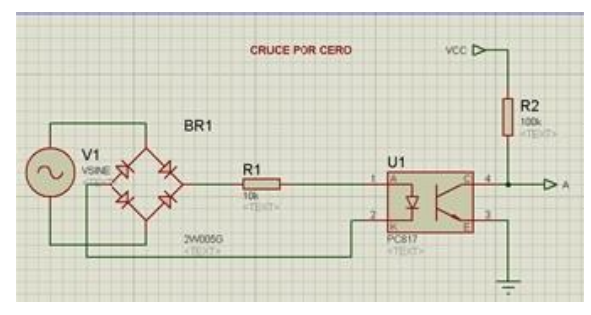

Figura 11. Forma de ondas generadas

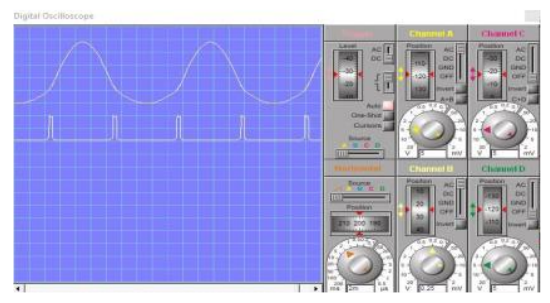

Fuente: Elaboración propia

Segunda Etapa: Referido al circuito integrador y seguidor de tensión elemento que permiten la tensión de salida, determinada por la longitud de tiempo de entrada, la señal DC que es acoplada a un transistor BC547, Obteniendo una onda de dientes de Sierra, dentro del diagrama del integrado y seguidor de la atención cómo se aprecia en la figura 12 y las ondas generadas en la figura 13 (Yaulema y Rosero 2016).

Figura 12 Diagrama circuito integrador

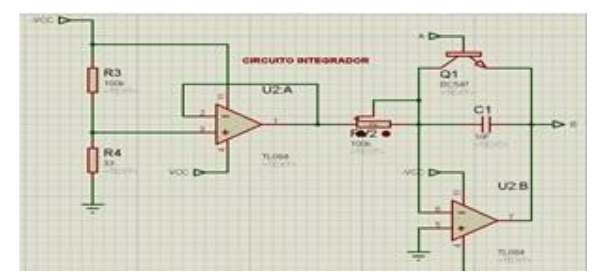

Figura 13 Señales generadas por el circuito Integrador

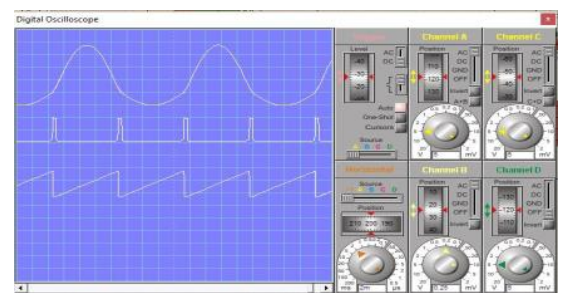

Fuente: Elaboración propia

Tercera Etapa: El circuito Restador -Inversor, resta e invierte la generación de la onda la cual permite eliminar el desfase establecido entre el voltaje de la señal de diente de Sierra la cual posee un mejor control del motor este circuito podemos observar en la figura $14 \mathrm{y} \mathrm{su}$ forma de onda en la figura 15 (Yaulema y Rosero 2016). 

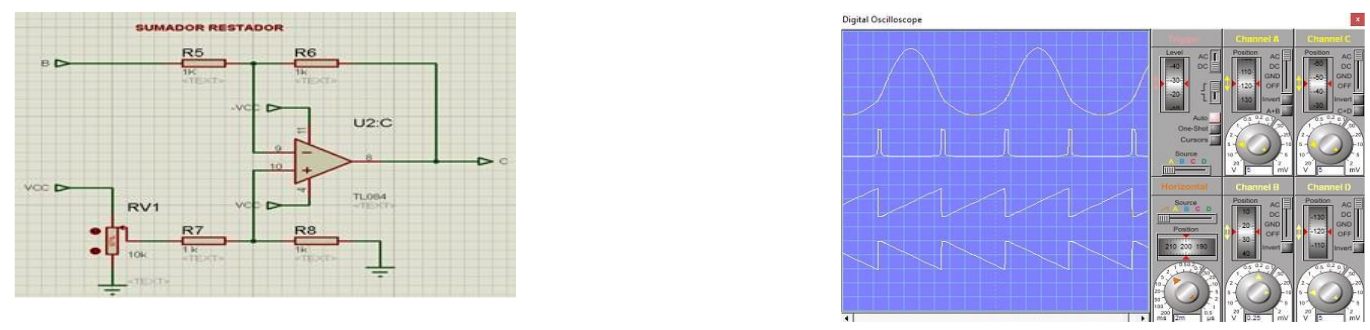

Fuente: Elaboración propia

Cuarta etapa: Comparador, dentro de este ciclo se realiza la comparación del voltaje tanto de la entrada y salida la cual clasifica la misma permitiendo el pase del mayor voltaje generando el reporte al PLC, utilizando para la simulación un potenciómetro el cual tiene la factibilidad de variar la señal de los pulsos observando la figura 16 y el tren de pulsos en la figura 17 (Yaulema y Rosero 2016).

Figura 16. Circuito de comparador de voltajes

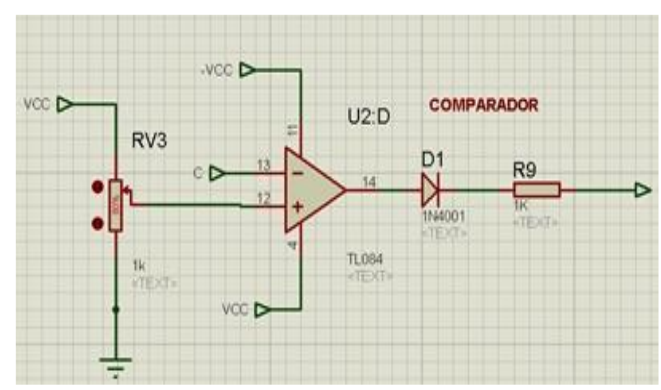

Figura 17 Señal generada por el comparador

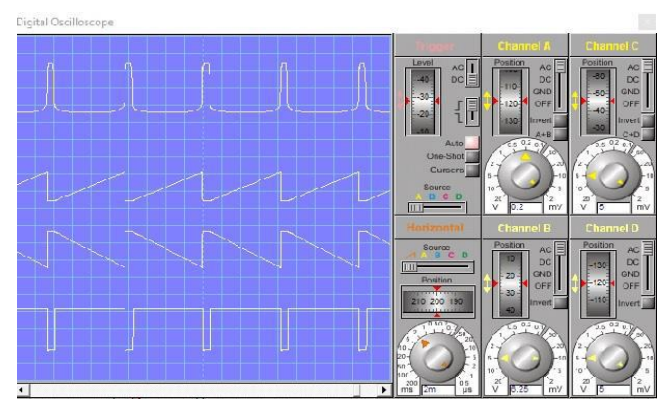

Fuente: Elaboración propia

Quinta etapa: circuito de potencia: La característica de esta fase es que se conoce conecta la salida del tren de pulsos a un optoacoplador con la finalidad de separar la parte qué roja baja potencia con la parte del AC, el cual al disparar el tristor, rectifica la forma de la onda ya sea a diferentes grados, el único condicionante es la señal de entrada la cual controla el motor y regula la intensidad cómo podemos observar en la figura 18 y la señal rectificada en la figura 19 (Yaulema y Rosero 2016). 
Figura 18 Diagrama circuito de potencia

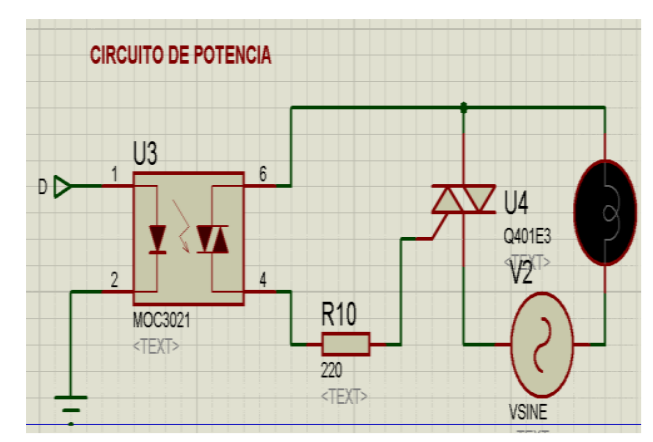

Figura 19 Señal de disparo del Tiristor

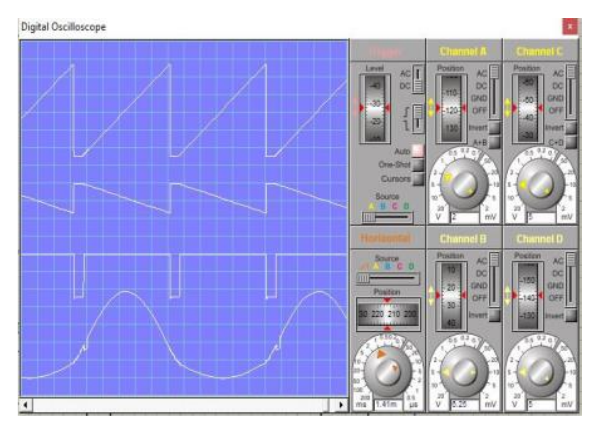

Fuente: Elaboración propia

Diseño driver para el motor a pasos del sistema monocromador: para la operatividad del motor se utilizó el círculo integrado L 298, constituido por un conductor doble corriente, interactuando por niveles lógicos TTL, el cual facilita el manejo de cargas inductivas como Relés, solenoides DC y motores paso a paso, diodos leds, para indicadores del encendido de las bobinas y los diodos rectificadores para la protección simplificándose este diagrama en la figura 20 (Yaulema y Rosero 2016).

Figura 20 Driver de Motor a Pasos de 8 cables

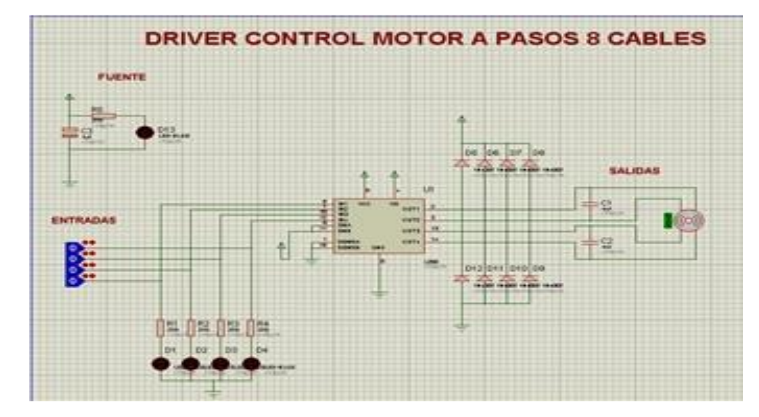

Fuente: Elaboración propia

Diseño del driver para el motor a pasos para la rendija: debido a las características del motor se usó un tip 122 este diseño lo podemos observar en la figura 21, generando un puente H qué tiene la capacidad de soportar corrientes mayores a 1 A. (Yaulema y Rosero 2016) 
Figura 21 Driver de Motor a Pasos de 6 cables

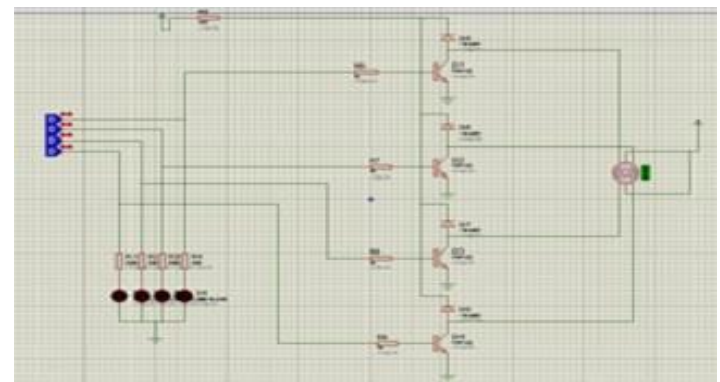

Fuente: Elaboración propia

Circuito PCB (Printed Circuit Board) para efectuar el control de motores: Una vez probado y simulado, Se realizó la validación y el diseño de los circuitos PCB, dentro del programa ARES, formica 3d de las siguientes estructuras, mostradas en la figura 22,23,24 (Yaulema y Rosero 2016).

Figura 22 Driver Motor AC

Figura 23 Driver Motor a Pasos 8 Figura 24 Driver Motor a Pasos 6
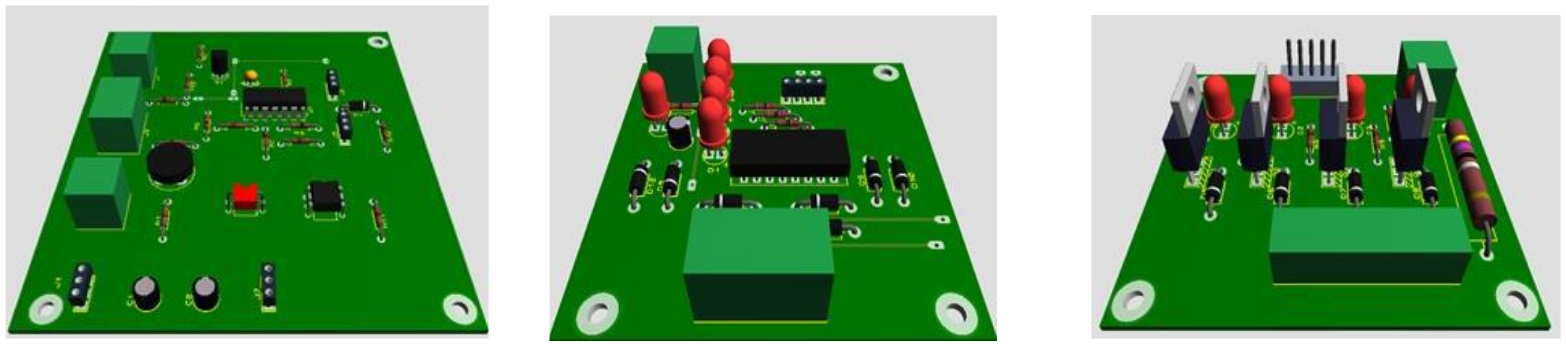

Fuente: Elaboración propia

Diseño de amplificación del voltaje ocupados en los optoacopladores de ranuras: utilizados para la detección de las ruedas y la posición del motor colocando dos optoacopladores de ranura, los cuales en su salida muestran un voltaje máximo de $5 \mathrm{~V}$, cómo podemos observar en la figura 25 (Yaulema y Rosero 2016).

Figura 25 Circuito de amplificación Opto acopladores

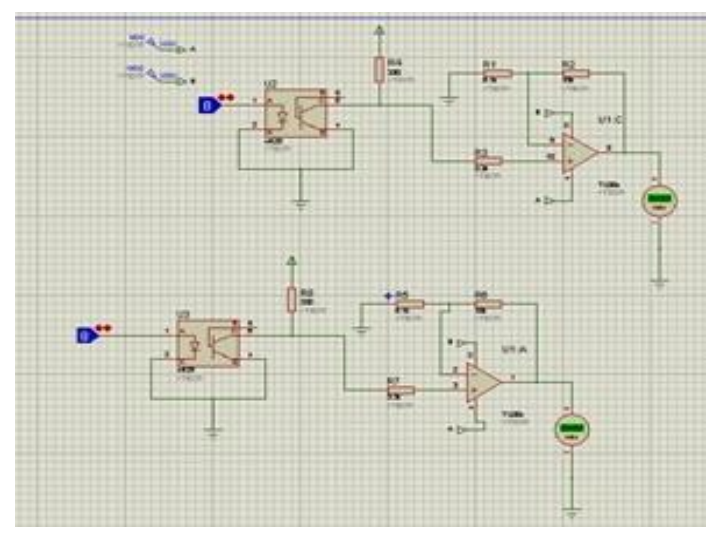

Fuente: Elaboración propia 
Diseño análisis y construcción de la base y la carcasa protectora: para la fabricación de la base se utilizó un material resistente el cual soporta todos los pesos de los componentes esta carcasa protectora tiene que ser especialmente de color oscuro (negro), cumpliendo con las funciones de evitar la contaminación con entrada de luz y salida de la misma, observadas en la figura 26 (Yaulema y Rosero 2016).

Figura. 26 Carcasa

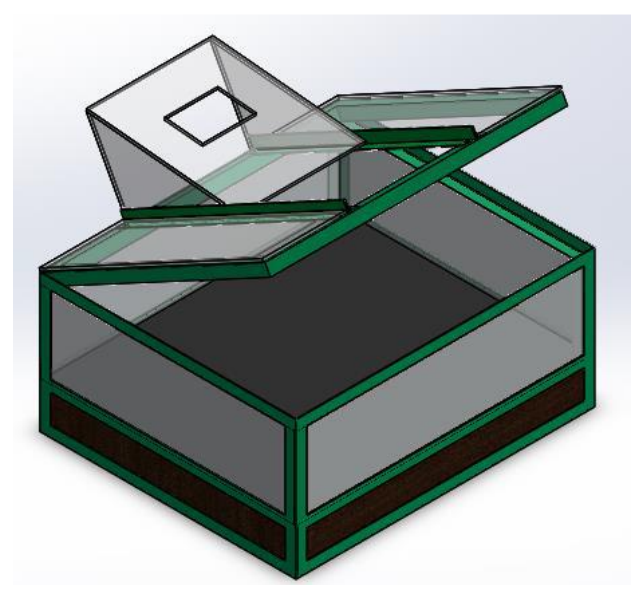

Fuente: Elaboración propia

Programación y funcionamiento: Al establecer y programar el PLC, se debe considerar todo el control de los actuadores para generar datos a través del HMI, y los sensores que funcionan dentro del sistema como podemos observar en la figura 27 (Yaulema y Rosero 2016).

Figura 27. Diagrama general del sistema

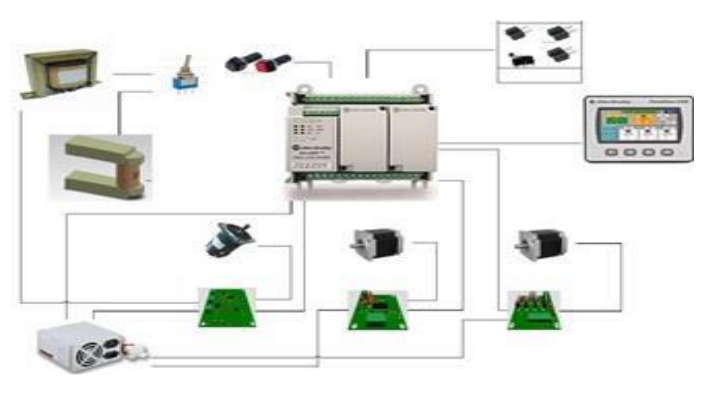

Fuente: Elaboración propia

Diseño de la interfaz Hombre máquina (HMI): el análisis diseño y programación del PLC, se lo realizó en el programa Connected Components Workbench, generando una interfaz de 
ISSN: 2600-5859

3 ventanas la carátula del menú principal y una pantalla de calibración de funcionamiento manual como observamos en las figuras 28,29 y30 . (Yaulema y Rosero 2016)

Figura 28. Presentación HMI

Figura 29. Ventana principal

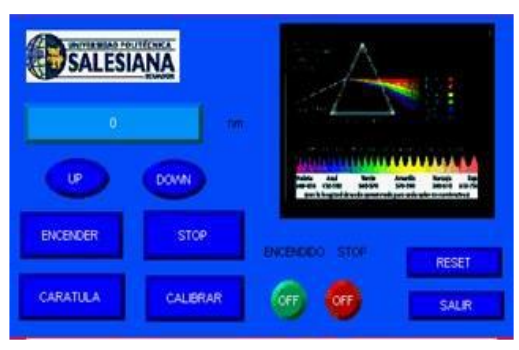

Fuente: Elaboración propia
Figura 30. Calibración del HMI

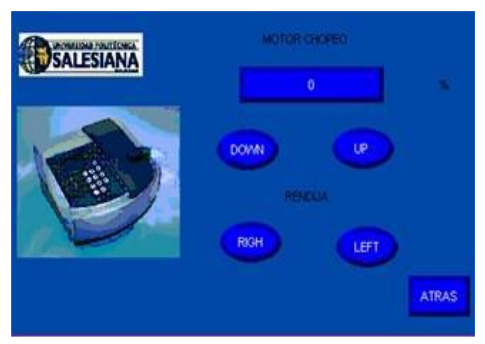

\section{Conclusiones.}

- El mecanismo propuesto para la generación de longitudes de onda UV, presenta un rediseño en su parte eléctrica y electrónica, manejados por varios actuadores con solo autómata cómo es la conexión de un HMI, poniendo a punto y funcionamiento los motores el encendido de la lámpara las protecciones eléctricas por microcontroladores a un PLC, otorgando un manejo óptimo del sistema.

- Para realizar la cuantificación de la longitud de onda se utilizaron varias fórmulas estadísticas generando un grado positivo de satisfacción al sistema, dentro de las cuales las dos opciones como son la interpolación de Newton, disminuyen el error al 0,05\% y la de mínimos cuadrado de $3.32 \%$, Generando valores óptimos de longitud de onda específicos para el tratamiento de las aguas residuales, comprobando la efectividad del sistema.

- Mediante la modificación del sistema mecánico del monocromador y rendija selectora se establecieron los valores de longitud de onda para poder visualizar y modificar por parte del usuario en una interfaz interactiva determinando los rangos operativos de la lámpara UV, comprendidos entre 500 y $700 \mathrm{~nm}$, valores óptimos para estudios de catálisis foto de graduación y descontaminación de aguas surfactantes y fenoles.

- El HMI, permite la visualización de la longitud de onda y el control de los motores facilitando y desarrollando una interacción directa entre el operador y el equipo, generando mejoras del sistema original en cuanto a sus actuadores y sensores con la obtención de 500 a 700 nm de longitud de onda, su sistema electrónico es característico en la protección de la lámpara, debido a su naturaleza endeble cuyo caso de aumentar los valores de corriente y voltaje preestablecidos, se puede generar un efecto flyback, dentro de la lámpara terminando así su vida útil. 


\section{Referencias Bibliográficas.}

Córdoba, U. (2009). Obtenido de Departamento de eléctrica. Disponible en: http://www.efn. uncor.edu/departamentos/electro/cat/eye_archivos/apuntes/a_practico/CAP\%209\%2 OPco.pdf

Elmer, P. (2004). Perkin Elmer. Obtenido de Lambda 650, 800, and 950, Spectrophotometers: Disponible en: https://www.perkinelmer.com/labsolutions//resources/docs/BRO_L ambda950850650Americas.pdf 8-12p.

Garcés, L., Mejía, E., \& Santamaría, J. (2011). Revista. Obtenido de Universidad Lasallista: Disponible en: http://www.lasallista.edu.co/fxcul/media/pdf/Revista/Vol1n1/08392\%20La\%20fotocat\%C3\%A1lisis\%20como\%20alternativa\%20para\%20el\%20trat amiento.pdf . 10-15p.

Granada, U. (2015). Espectroscopia. Obtenido de Espectroscopia Ultravioleta visible. Disponible en: http://www.ugr.es/ quiored/espec/uv.htm

Harris, D. (2006). Análisis Químico Cuantitativo. New York: Reverte.

Paullan, D. (2015). Degradación del fenol presente en las descargas de aguas residuos industriales Shushufindi mediante fotoxidación catalítica homogénea y heterogénea descontaminación de descargas. Riobamba: ESPOCH. 15 -16 p.

Pérez, D., y Rengifo, J. (2011). villamor. Obtenido de espejosplanosyesfericos: http://villamorespejosplanosyesfericos.blogspot.com/

Peri, C. (2015). Instituto Argentino de Radioastronomía. Disponible en: http://www.iar.unlp.edu.ar/divulgacion/art-difu-21.htm

Requena, A., y Zúñiga, J. (2004). Espectroscopía. Murcia: Pearson. 12-17p

RockwellAutomation. (2014). rockwellautomation. Obtenido de iterature/documents: http://literature.rockwellautomation.com/idc/groups/literature/documents/br/2080br001_-es-p.pdf

Sirtori, C., López, A., \& Rodríguez, S. (2010). Evaluación analítica de procesos de transformación biológica, fotoquímica y fotocatalítica de fármacos en agua. Editorial Ciemat.

Skoog, D. A. (2008). Principios Análisis Instrumental. México: CENGAGE.

Skoog, D., Holler, F., \& Crouch, S. (2008). Principios de análisis Instrumental. México: CENGAGE. 
ISSN: 2600-5859

Vol. 3, $\mathrm{N}^{\circ} 2.2$, p. 132-153, junio, 2020

Todorobot. (2016). Todorobot. Obtenido de motores, Disponible en: http://www.todorobot.com.ar/tutorial-sobre-motores-paso-a-paso-stepper-motors/

Viticodoc. (2010). Scribd. Campos visuales normales. Disponible en: http://es.scribd.com/doc/35067879/FISICA-Y-OPTICA\#scribd

Yaulema, J., y Rosero, J., (2016). Automatización de un generador de luz ultravioeta visible de longitud de onda variable, controlado con un HMI, (Tesis Pregrado).Quito. Universidad Salesiana.

Zúñiga, A. R. (2004). Espectroscopía. En A. R. Zuñiga, Espectroscopía. Murcia: Pearson. $39 \mathrm{p}$.

\section{PARA CITAR EL ARTÍCULO INDEXADO.}

Yaulema Castañeda, J. L., Bolaños Logroño, P. F., Mendez Gómez, H. B., \& Orquera, V. (2020). Automatización de un generador de luz ultravioleta, controlada con un $\mathrm{HMI}$, de longitud de onda variable. ConcienciaDigital, 3(2.2), 132-153. https://doi.org/10.33262/concienciadigital.v3i2.2.1252

\section{LCiencia}

El artículo que se publica es de exclusiva responsabilidad de los autores y no necesariamente reflejan el pensamiento de la Revista Conciencia Digital.

El artículo queda en propiedad de la revista y, por tanto, su publicación parcial y/o total en otro medio tiene que ser autorizado por el director de la Revista Conciencia Digital.

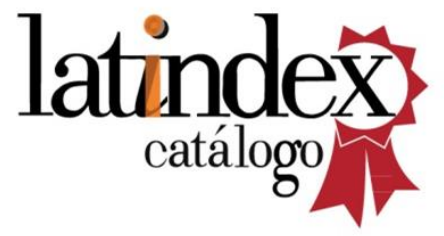

Conciencia

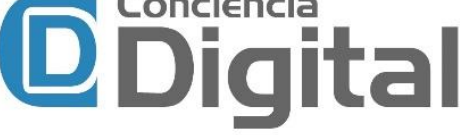

